\title{
Stratifications on the Moduli Space of Higgs Bundles
}

\author{
$02 / 11 / 2016$ \\ Peter B. Gothen 1 \\ Centro de Matemática da Universidade do Porto CMUP \\ Faculdade de Ciências da Universidade do Porto FCUP \\ Rua do Campo Alegre, s/n \\ 4197-007 Porto, Portugal \\ e-mail: pbgothen@fc.up.pt \\ Ronald A. Zúñiga-Rojaঐ2 \\ Centro de Investigaciones Matemáticas y Metamatemáticas CIMM \\ Universidad de Costa Rica UCR \\ San José 11501, Costa Rica \\ e-mail: ronald.zunigarojas@ucr.ac.cr
}

\begin{abstract}
The moduli space of Higgs bundles has two stratifications. The BiałynickiBirula stratification comes from the action of the non-zero complex numbers by multiplication on the Higgs field, and the Shatz stratification arises from the Harder-Narasimhan type of the vector bundle underlying a Higgs bundle. While these two stratifications coincide in the case of rank two Higgs bundles, this is not the case in higher rank. In this paper we analyze the relation between the two stratifications for the moduli space of rank three Higgs bundles.
\end{abstract}

Keywords: Moduli of Higgs Bundles, Harder-Narasimhan filtrations, Hodge Bundles, Vector Bundles. MSC class: Primary 14H60; Secondary 14 D07.

\section{Introduction}

Higgs bundles and their moduli were first studied by Hitchin and Simpson and have been around for almost 30 years. They continue to be the subject of intensive investigations with links to diverse areas of mathematics such as non-abelian Hodge theory, integrable systems, mirror symmetry, the Langlands programme, among others.

In this paper we focus on the moduli space of Higgs bundles on a compact Riemann surface $X$. The topology of this moduli space has been studied extensively. Some early calculations of Betti numbers were carried out by Hitchin [19] for rank 2 and the first author [8] for rank 3. Further significant progress has been made by a number authors, see, e.g., [17, 18, 21, 11, 22, 15, 5, 14, 13, 10, 11]. Recently Schiffmann [25] has completely determined the additive cohomology in the case of Higgs bundles with rank and degree co-prime.

\footnotetext{
${ }^{1}$ Partially supported by CMUP (UID/MAT/00144/2013) and the projects PTDC/MATGEO/0675/2012 and PTDC/MAT-GEO/2823/2014, funded by FCT (Portugal) with national and, where applicable, European structural funds through the programme FEDER, under the partnership agreement PT2020.

${ }^{2}$ Supported by Universidad de Costa Rica through CIMM (Centro de Investigaciones Matemáticas y Metamatemáticas), through the Project 820-B5-202. Partially supported by FCT (Portugal) through grant SFRH/BD/51174/2010.
} 
On the other hand, the homotopy theory of the moduli space of Higgs bundles has not been the subject of a lot of interest. Hausel [12] in his thesis studied the case of rank 2 Higgs bundles, while in [4] some results were obtained for general rank. The latter paper used the Białynicki-Birula stratification of the Higgs bundle moduli space coming from the $\mathbb{C}^{*}$-action given by multiplying the Higgs field by scalars. In rank 2 this stratification coincides with the Shatz stratification, which is given by the Harder-Narasimhan type of the vector bundle underlying a Higgs bundle. As already observed by Hitchin and exploited by Hausel and Thaddeus [12, 17] this makes the case of rank 2 Higgs bundles akin to a finite dimensional version of the infinite dimensional situation of Atiyah-Bott [2].

However, in general the Białynicki-Birula and Shatz stratifications do not coincide, and it is therefore of interest to study their relationship. In this paper we carry out such a study in the case of rank 3 Higgs bundles, where it turns out that the situation is already fairly complicated. Indeed, our main result, Theorem 5.1, shows that each Shatz stratum is intersected by several different Białynicki-Birula strata. Moreover, knowledge of the underlying vector bundle of a Higgs bundle is not sufficient to determine its BiałynickiBirula stratum, one also needs knowledge of the Higgs field. However, for sufficiently unstable underlying vector bundles the situation is simpler and the Shatz strata coincide with Białynicki-Birula strata: this is described in Theorem 5.6.

Our results should serve as a useful pointer to the general situation for higher rank Higgs bundles. Moreover, in the aforementioned work [12, 17], Hausel and Thaddeus consider the moduli space of $k$-Higgs bundles (where the Higgs field is allowed to have a pole of order $k$ at a fixed $p \in X$ ), and show that in the limit $k \rightarrow \infty$ this moduli space approximates the classifying space of the gauge group. This is used by Hausel [12, Theorem 7.5.7] in the rank two case to calculate certain homotopy groups of the moduli space of Higgs bundles, using implicitly that the Białynicki-Birula and Shatz stratifications coincide. One might thus hope that an extension of our results to Higgs bundles with poles could be useful in extending Hausel's results to higher rank.

This paper is organized as follows. In Section 2 we give some preliminaries about Higgs bundles and their moduli spaces and we explain the Białynicki-Birula and Shatz stratifications of the moduli space. Next, for completeness, in Section 3 we present the aforementioned result of Hausel on the equality of the two stratifications for rank 2 Higgs bundles. After that, in Section 4, we give some bounds on the Harder-Narasimhan types which occur in the moduli space of rank 3 Higgs bundles. Finally, in Section 5, we give our main results on the relation of the two stratifications.

This paper is partly based on the Ph.D. thesis [28] of the second author and an announcement of some of our results has appeared in [27].

\section{Preliminaries}

\subsection{Higgs bundles and their moduli}

Let $X$ be a closed Riemann surface of genus $g$ and let $K=K_{X}=T^{*} X$ be the canonical line bundle of $X$.

Definition 2.1. A Higgs bundle over $X$ is a pair $(E, \Phi)$ where the underlying vector bundle $E \rightarrow X$ is a holomorphic vector bundle and the Higgs field $\Phi: E \rightarrow E \otimes K$ is a holomorphic endomorphism of $E$ twisted by $K$. 
The slope of a vector bundle $E$ is the quotient between its degree and its rank: $\mu(E)=$ $\operatorname{deg}(E) / \operatorname{rk}(E)$. Recall that a vector bundle $E$ is semistable if $\mu(F) \leqslant \mu(E)$ for all non-zero subbundles $F \subset E$, stable if it is semistable and strict inequality holds for all non-zero proper $F$, and polystable if it is the direct sum of stable bundles, all of the same slope. Any semistable vector bundle has a Jordan-Hölder filtration $E_{0} \subset E_{1} \subset \cdots \subset E$ such that the subquotients $E_{j} / E_{j-1}$ are stable. The isomorphism class of the associated graded bundle $\oplus E_{j} / E_{j-1}$ is unique, and semistable vector bundles are $S$-equivalent if their associated graded bundles are isomorphic. Each $S$-equivalence class contains a unique polystable representative. The corresponding notions for Higgs bundles are defined in exactly the same way, except that only $\Phi$-invariant subbundles $F \subset E$ (satisfying $\Phi(F) \subset F \otimes K$ ) are considered in the stability conditions.

The moduli space $\mathcal{M}(r, d)$ of $S$-equivalence classes of semistable rank $r$ and degree $d$ Higgs bundles was constructed by Nitsure [23]. The points of $\mathcal{M}(r, d)$ correspond to isomorphism classes of polystable Higgs bundles. When $r$ and $d$ are co-prime any semistable Higgs bundle is automatically stable and $\mathcal{M}(r, d)$ is smooth.

There are no stable Higgs bundles when $g \leqslant 1$ and the theory has quite a different flavour (see, for example, the work of Franco-García-Prada-Newstead [6, 7] on Higgs bundles on elliptic curves), and so we shall also assume that $g \geqslant 2$.

We shall need to consider the moduli space from the complex analytic point of view. For this, fix a complex $C^{\infty}$ vector bundle $\mathcal{E}$ of rank $r$ and degree $d$ on $X$. A holomorphic structure on $\mathcal{E}$ is given by a $\bar{\partial}$-operator $\bar{\partial}_{E}: A^{0}(\mathcal{E}) \rightarrow A^{0,1}(\mathcal{E})$ and we thus obtain a holomorphic vector bundle $E=\left(\mathcal{E}, \bar{\partial}_{E}\right)$. From this point of view, a Higgs bundle $(E, \Phi)$ arises from a pair $\left(\bar{\partial}_{E}, \Phi\right)$ consisting of a $\bar{\partial}$-operator and a Higgs field $\Phi \in A^{1,0}(\operatorname{End}(\mathcal{E}))$ such that $\bar{\partial}_{E} \Phi=0$. The natural symmetry group of the situation is the complex gauge group $\mathcal{G}^{\mathbb{C}}=\left\{g: \mathcal{E} \rightarrow \mathcal{E} \mid g\right.$ is a $C^{\infty}$ bundle isomorphism $\}$, which acts on pairs $\left(\bar{\partial}_{E}, \Phi\right)$ in the standard way:

$$
g \cdot\left(\bar{\partial}_{E}, \Phi\right)=\left(g \circ \bar{\partial}_{E} \circ g^{-1}, g \circ \Phi \circ g^{-1}\right) .
$$

The moduli space can then be viewed as the quotient 3

$$
\mathcal{M}(r, d)=\left\{\left(\bar{\partial}_{E}, \Phi\right) \mid \bar{\partial}_{E} \Phi=0 \text { and }(E, \Phi) \text { is polystable }\right\} / \mathcal{G}^{\mathbb{C}} .
$$

\subsection{Harder-Narasimhan filtrations and the Shatz stratification}

The Harder-Narasimhan filtration of a vector bundle was introduced in [9, Proposition 1.3.9] and studied systematically by Shatz [24, Section 3]. It plays an important role in the work of Atiyah and Bott [2, Section 7]. We refer the reader to these references for details on what follows.

Let $E$ be a holomorphic vector bundle on $X$. A Harder-Narasimhan Filtration of $E$, is a filtration of the form

$$
\operatorname{HNF}(E): E=E_{s} \supset E_{s-1} \supset \cdots \supset E_{1} \supset E_{0}=0
$$

which satisfies the following two properties:

(i) $\mu\left(E_{j+1} / E_{j}\right)<\mu\left(E_{j} / E_{j-1}\right)$ for $1 \leqslant j \leqslant s-1$.

\footnotetext{
${ }^{3}$ Strictly speaking one should use appropriate Sobolev completions as in Atiyah and Bott [2, Section 14]; see, for example, Hausel and Thaddeus [17. Section 8] for the case of Higgs bundles.
} 
(ii) $E_{j} / E_{j-1}$ is semistable for $1 \leqslant j \leqslant s$.

For brevity, when we have a filtration $E=E_{s} \supset E_{s-1} \supset \cdots \supset E_{1} \supset E_{0}=0$ we shall sometimes write $\bar{E}_{j}=E_{j} / E_{j-1}$ for the subquotients. The associated graded vector bundle is

$$
\operatorname{Gr}(E)=\bigoplus_{j=1}^{s} E_{j} / E_{j-1}=\bigoplus_{j=1}^{s} \bar{E}_{j} .
$$

Any vector bundle $E$ has a unique Harder-Narasimhan filtration. The subbundle $E_{1} \subset E$ is called the maximal destabilizing subbundle of $E$; its rank is maximal among subbundles of $E$ of maximal slope. Consider the Harder-Narasimhan polygon as the polygon in the $(r, d)$-plane with vertices $\left(\operatorname{rk}\left(E_{j}\right), \operatorname{deg}\left(E_{j}\right)\right)$ for $j=0, \ldots, s$. The slope of the line joining $\left(\operatorname{rk}\left(E_{j-1}\right), \operatorname{deg}\left(E_{j-1}\right)\right)$ and $\left(\operatorname{rk}\left(E_{j}\right), \operatorname{deg}\left(E_{j}\right)\right)$ is $\mu\left(\bar{E}_{j}\right)$. Condition (i) above says that the Harder-Narasimhan polygon is convex. Clearly this is equivalent to saying that $\mu\left(E_{j}\right)<\mu\left(E_{j-1}\right)$ for $j=2, \ldots, s$.

The Harder-Narasimhan type of $E$ is the following vector in $\mathbb{R}^{r}$ :

$$
\operatorname{HNT}(E)=\mu=\left(\mu\left(\bar{E}_{1}\right), \ldots, \mu\left(\bar{E}_{1}\right), \ldots, \mu\left(\bar{E}_{s}\right), \ldots, \mu\left(\bar{E}_{s}\right)\right)
$$

where $r=\operatorname{rk}(E)$, and the slope of each $\bar{E}_{j}$ is repeated $r_{j}=\operatorname{rk}\left(\bar{E}_{j}\right)$ times.

There is a finite decomposition of $\mathcal{M}(r, d)$ by the Harder-Narasimhan type of the underlying vector bundle $E$ of a $\operatorname{Higgs}$ bundle $(E, \Phi)$ :

$$
\mathcal{M}(r, d)=\bigcup_{\mu} U_{\mu}^{\prime}
$$

where $U_{\mu}^{\prime} \subset \mathcal{M}(r, d)$ is the subspace of Higgs bundles $(E, \Phi)$ whose underlying vector bundle $E$ has Harder-Narasimhan type $\mu$. When $(E, \Phi)$ is strictly semistable we take its Harder-Narasimhan type to be that of the polystable representative of its $S$-equivalence class. As a consequence of Shatz [24, Propositions 10 and 11] the decomposition (2.2) has nice properties and for this reason it is known as the Shatz stratification. Note that there is an open dense stratum $U_{(d / r, \ldots, d / r)}^{\prime}$ corresponding to Higgs bundles $(E, \Phi)$ for which the underlying vector bundle $E$ is itself semistable (see Hitchin[20, Proposition 6.1] in the rank 2 case and [4, Proposition 3.12] for general rank). Since $\Phi \in H^{0}(\operatorname{End}(E) \otimes K) \cong$ $H^{1}(\operatorname{End}(E))^{*}$ (by Serre duality), such a Higgs bundle represents a point in the cotangent bundle of the moduli space of stable bundles $\mathcal{N}^{s}(r, d)$ when $E$ is stable. Thus, if $(r, d)=1$

$$
U_{(d / r, \ldots, d / r)}^{\prime}=T^{*} \mathcal{N}(r, d) \subset \mathcal{M}(r, d) .
$$

\subsection{The $\mathbb{C}^{*}$-action and the Białynicki-Birula stratification}

We review some standard facts about the $\mathbb{C}^{*}$-action on $\mathcal{M}(r, d)$. For more details see, e.g., Simpson [26, Section 4], especially Lemma (4.1.).

The holomorphic action of the multiplicative group $\mathbb{C}^{*}$ on $\mathcal{M}(r, d)$ is defined by the multiplication:

$$
z \cdot(E, \Phi) \mapsto(E, z \cdot \Phi)
$$

The limit $\left(E_{0}, \varphi_{0}\right)=\lim _{z \rightarrow 0} z \cdot(E, \Phi)$ exists for all $(E, \Phi) \in \mathcal{M}(r, d)$. Moreover, this limit is fixed by the $\mathbb{C}^{*}$-action. A Higgs bundle $(E, \Phi)$ is a fixed point of the $\mathbb{C}^{*}$-action if and only if it is a Hodge bundle, i.e. there is a decomposition $E=\bigoplus_{j=1}^{p} E_{j}$ with respect to which the Higgs field has weight one: $\Phi: E_{j} \rightarrow E_{j+1} \otimes K$. The type of the Hodge bundle $(E, \Phi)$ is $\left(\operatorname{rk}\left(E_{1}\right), \ldots, \operatorname{rk}\left(E_{p}\right)\right)$. 
Let $\left\{F_{\lambda}\right\}$ be the irreducible components of the fixed point locus of $\mathbb{C}^{*}$ on $\mathcal{M}(r, d)$. Let

$$
U_{\lambda}^{+}:=\left\{(E, \Phi) \in \mathcal{M} \mid \lim _{z \rightarrow 0} z \cdot(E, \Phi) \in F_{\lambda}\right\} .
$$

Then we have the Biatynicki-Birula stratification (cf. [3, Theorem 4.1]) of $\mathcal{M}(r, d)$ :

$$
\mathcal{M}=\bigcup_{\lambda} U_{\lambda}^{+}
$$

Note that there is a distinguished component

$$
F_{\min }=\mathcal{N}(r, d)
$$

of the fixed locus corresponding to semistable Higgs bundles with zero Higgs field and that we have a corresponding Białynicki-Birula stratum $U_{\min }^{+}$. Let $(E, \Phi)$ be a semistable Higgs bundle. When the underlying vector bundle $E$ is itself semistable, clearly $\lim _{z \rightarrow 0} z$. $(E, \Phi)=(E, 0)$. Conversely, if $\lim _{z \rightarrow 0} z \cdot(E, \Phi)=(E, 0) \in \mathcal{M}(r, d)$, then $(E, 0)$ is a semistable Higgs bundle and hence $E$ is a semistable vector bundle. Thus we have the following result, valid for any rank.

Proposition 2.2. Let $(E, \Phi) \in \mathcal{M}(r, d)$. Then $\lim _{z \rightarrow 0} z \cdot(E, \Phi)=(E, 0)$ if and only if E is semistable.

In view of this result the following proposition is now immediate.

Proposition 2.3. The following subspaces of the moduli space $\mathcal{M}(r, d)$ coincide:

$$
U_{(d / r, \ldots, d / r)}^{\prime}=U_{\min }^{+} .
$$

\section{The rank 2 case}

In this section we recall, for completeness, a theorem of Hausel, which says that in rank 2 the Shatz and Białynicki-Birula stratifications coincide.

Let $(E, \Phi)$ be a semistable rank 2 Higgs bundle corresponding to a fixed point of the $\mathbb{C}^{*}$-action on $\mathcal{M}(2, d)$. In view of the results explained in Section 2.3 , either $\Phi=0$ or $(E, \Phi)$ is of the form

$$
(E, \Phi)=\left(E_{1} \oplus E_{2},\left(\begin{array}{cc}
0 & 0 \\
\varphi & 0
\end{array}\right)\right)
$$

Let $d_{1}=\operatorname{deg}\left(E_{1}\right)$, then $\operatorname{deg}\left(E_{2}\right)=d-d_{1}$. Semistability of $(E, \Phi)$ immediately shows that $d_{1}$ must satisfy the bounds

$$
d \leqslant 2 d_{1} \leqslant d+2 g-2 .
$$

If $d<2 d_{1}$ then $\varphi \neq 0$, and if $d=2 d_{1}$ then such a Higgs bundle is $S$-equivalent to $(E, 0)$. Thus, the components of the fixed locus are $F_{\min }=\mathcal{N}(2, d)$ and, for each $d_{1}$ with $d<2 d_{1} \leqslant d+2 g-2$, a component $F_{d_{1}}$ consisting of $(E, \Phi)$ of the form (3.1). (It is easy to see that $F_{d_{1}}$ is indeed connected, cf. Hitchin [19, Sec. 7].)

The methods employed in the present paper readily give the following result (cf. Remark 5.7). 
Proposition 3.1. Let $(E, \Phi) \in \mathcal{M}(2, d)$ be a rank 2 Higgs bundle such that $E$ is an unstable vector bundle with maximal destabilizing line bundle $E_{1} \subset E$. Then the limit $\left(E_{0}, \Phi_{0}\right)=\lim _{z \rightarrow 0}(E, z \cdot \Phi)$ is

$$
\left(E_{0}, \Phi_{0}\right)=\left(E_{1} \oplus E / E_{1},\left(\begin{array}{cc}
0 & 0 \\
\varphi_{21} & 0
\end{array}\right)\right)
$$

where $\varphi_{21}$ is induced from $\Phi$. The associated graded vector bundle is $\operatorname{Gr}\left(E_{0}\right)=\operatorname{Gr}(E)$.

Combining Proposition 3.1 with Proposition 2.2 immediately gives the following corollary.

Corollary 3.2 (Hausel [12, Proposition 4.3.2]). The Shatz stratification of $\mathcal{M}(2, d)$ coincides with the Biatynicki-Birula stratification. More precisely, $U_{(d / 2, d / 2)}^{\prime}=U_{\min }^{+}=$ $T^{*} \mathcal{N}(2, d)$ (where the last identity holds for $d$ odd), and $U_{d_{1}, d-d_{1}}^{\prime}=U_{d_{1}}^{+}$for each $d_{1}$ satisfying $d<2 d_{1} \leqslant d+2 g-2$.

\section{Bounds on Harder-Narasimhan types in rank 3}

Let $(E, \Phi)$ be a rank 3 Higgs bundle. Let $\left(\mu_{1}, \mu_{2}, \mu_{3}\right)$ be the Harder-Narasimhan type of $E$, so that $\mu_{1} \geqslant \mu_{2} \geqslant \mu_{3}$ and $\mu_{1}+\mu_{2}+\mu_{3}=3 \mu$, where $\mu=\mu(E)$. We can write the Harder-Narasimhan filtration of the vector bundle $E$ as follows:

$$
\operatorname{HNF}(E): 0=E_{0} \subset E_{1} \subset E_{2} \subset E_{3}=E,
$$

where we have made the convention that $E_{i}=E_{j}$ if $\mu_{i}=\mu_{j}$. Thus, for example, if $\mu_{1}=\mu_{2}>\mu_{3}$ then the Harder-Narasimhan filtration is

$$
\operatorname{HNF}(E): 0=E_{0} \subset E_{1}=E_{2} \subset E_{3}=E
$$

and $\operatorname{rk}\left(E_{1}\right)=\operatorname{rk}\left(E_{2}\right)=2$. Similarly, if $\mu_{1}>\mu_{2}=\mu_{3}$ then $\operatorname{rk}\left(E_{1}\right)=1$ and $\operatorname{rk}\left(E_{2}\right)=3$.

We shall next introduce some notation which will be used throughout the remainder of the paper.

Let $\varphi_{21}: E_{1} \rightarrow E / E_{1} \otimes K$ be the map induced by $\Phi$ and let

$$
I \subset E / E_{1}
$$

be the subbundle defined by saturating the subsheaf $\varphi_{21}\left(E_{1}\right) \otimes K^{-1} \subset E / E_{1}$. Similarly, let $\varphi_{32}: E_{2} \rightarrow E / E_{2} \otimes K$ be the map induced by $\Phi$ and let

$$
N=\operatorname{ker}\left(\varphi_{32}\right) \subset E_{2}
$$

viewed as a subbundle.

Remark 4.1. Let $(E, \Phi)$ be a stable Higgs bundle such that $E$ is an unstable vector bundle of Harder-Narasimhan type $\left(\mu_{1}, \mu_{2}, \mu_{3}\right)$. Then $E_{1} \subset E$ is destabilizing and hence, by stability of $(E, \Phi)$, we have $\varphi_{21} \neq 0$. Similarly $E_{2} \subset E$ is destabilizing and so $\varphi_{32} \neq 0$ (unless $\mu_{2}=\mu_{3} \Longleftrightarrow E_{2}=E$ ).

Proposition 4.2. Let $(E, \Phi)$ be a semistable rank 3 Higgs bundle of Harder-Narasimhan type $\left(\mu_{1}, \mu_{2}, \mu_{3}\right)$. Then

$$
\begin{aligned}
& 0 \leqslant \mu_{1}-\mu_{2} \leqslant 2 g-2, \\
& 0 \leqslant \mu_{2}-\mu_{3} \leqslant 2 g-2 .
\end{aligned}
$$


Proof. The fact that the differences $\mu_{i+1}-\mu_{i}$ are non-negative is just the convexity of the Harder-Narasimhan polygon.

If $E$ is semistable the result is clear, so we may assume that this is not the case.

If $\mu_{1}>\mu_{2}$ then $\operatorname{rk}\left(E_{1}\right)=1$, and $I \subset E / E_{1}$ is a line bundle, since $\varphi_{21} \neq 0$ by Remark 4.1. It follows that we have a non-zero map of line bundles $E_{1} \rightarrow I \otimes K$ and so

$$
\mu(I)+2 g-2 \geqslant \mu\left(E_{1}\right)=\mu_{1} .
$$

Also, since $E_{2} / E_{1} \subset E / E_{1}$ is the maximal destabilizing subbundle, we have that

$$
\mu(I) \leqslant \mu\left(E_{2} / E_{1}\right)=\mu_{2}
$$

(note that this inequality also holds if $\mu_{2}=\mu_{3}$ ). Combining these two inequalities proves (4.3).

If $\mu_{2}>\mu_{3}$ then $\operatorname{rk}\left(E_{2}\right)=2$, and $N \subset E_{2}$ is a line bundle, since $\varphi_{32} \neq 0$ by Remark 4.1. It follows that we have a non-zero map of line bundles $E_{2} / N \rightarrow E / E_{2} \otimes K$ and so

$$
\begin{aligned}
& \mu\left(E / E_{2}\right)+2 g-2 \geqslant \mu\left(E_{2} / N\right) \\
& \Longleftrightarrow \mu_{3}+2 g-2 \geqslant \operatorname{deg}\left(E_{2}\right)-\mu(N)=\mu_{1}+\mu_{2}-\mu(N) .
\end{aligned}
$$

Also, since $E_{1} \subset E_{2}$ is maximal destabilizing, we have that

$$
\mu(N) \leqslant \mu\left(E_{1}\right)=\mu_{1}
$$

(note that this inequality also holds if $\mu_{1}=\mu_{2}$ ). Combining these two inequalities proves (4.4).

Note that the proof of the preceding Proposition gives the following bounds on the slopes of the bundles $I$ and $N$.

Proposition 4.3. Let $(E, \Phi)$ be a semistable rank 3 Higgs bundle of Harder-Narasimhan type $\left(\mu_{1}, \mu_{2}, \mu_{3}\right)$ and define $I \subset E / E_{1}$ and $N \subset E_{2}$ as above.

(1) If $\mu_{1}>\mu_{2}$ then $I \subset E / E_{1}$ is a line subbundle of a rank 2 bundle and $\mu_{1}-(2 g-2) \leqslant$ $\mu(I) \leqslant \mu_{2}$.

(2) If $\mu_{2}>\mu_{3}$ then $N \subset E_{2}$ is a line subbundle of a rank 2 bundle and $\mu_{1}+\mu_{2}-\mu_{3}-$ $(2 g-2) \leqslant \mu(N) \leqslant \mu_{1}$.

\section{Limits of the $\mathbb{C}^{*}$-action}

The purpose of the present section is to analyse the limit as $z \rightarrow 0$ of $z \cdot(E, \Phi)$ as a function of the Harder-Narasimhan type of $E$. Note that the case of trivial HarderNarasimhan filtration, corresponding to $(E, \Phi)$ with semistable underlying vector bundle $E$, is covered by Proposition 2.2. 


\subsection{Non-trivial Harder-Narasimhan filtrations}

Again we limit ourselves to considering rank 3 stable Higgs bundles $(E, \Phi)$. We shall use the notation introduced in Section 4 .

Theorem 5.1. Let $(E, \Phi) \in \mathcal{M}(3, d)$ be such that $E$ is an unstable vector bundle of slope $\mu$ and with Harder-Narasimhan type $\left(\mu_{1}, \mu_{2}, \mu_{3}\right)$. Then the limit $\left(E_{0}, \Phi_{0}\right)=\lim _{z \rightarrow 0}(E, z \cdot \Phi)$ is given as follows.

(1) Assume that $\mu_{2}<\mu$. Then $\mu_{1}>\mu_{2} \geqslant \mu_{3}$, the subbundle $I \subset E / E_{1}$ defined in (4.1) is a line bundle, and one of the following alternatives holds.

(1.1) The slope of $I$ satisfies $\mu_{1}-(2 g-2) \leqslant \mu(I)<-\frac{1}{3} \mu_{1}+\frac{2}{3} \mu_{2}+\frac{2}{3} \mu_{3}$. Then $\left(E_{0}, \Phi_{0}\right)$ is the following Hodge bundle of type $(1,2)$ :

$$
\left(E_{0}, \Phi_{0}\right)=\left(E_{1} \oplus E / E_{1},\left(\begin{array}{cc}
0 & 0 \\
\varphi_{21} & 0
\end{array}\right)\right)
$$

where $\varphi_{21}$ is induced from $\Phi$. The associated graded vector bundle is $\operatorname{Gr}\left(E_{0}\right)=$ $\operatorname{Gr}(E)$.

(1.2) The slope of I satisfies $\mu(I)=-\frac{1}{3} \mu_{1}+\frac{2}{3} \mu_{2}+\frac{2}{3} \mu_{3}$. Then $\left(E_{0}, \Phi_{0}\right)$ is the following strictly polystable Hodge bundle:

$$
\left(E_{0}, \Phi_{0}\right)=\left(E_{1} \oplus I,\left(\begin{array}{cc}
0 & 0 \\
\varphi_{21} & 0
\end{array}\right)\right) \oplus\left(\left(E / E_{1}\right) / I, 0\right)
$$

where $\varphi_{21}$ is induced from $\Phi$. The associated graded vector bundle is $E_{0}=$ $\operatorname{Gr}\left(E_{0}\right)=E_{1} \oplus\left(E / E_{1}\right) / I \oplus I$ and its Harder-Narasimhan type is $\operatorname{HNT}\left(E_{0}\right)=$ $\left(\mu_{1}, \mu,-\frac{1}{3} \mu_{1}+\frac{2}{3} \mu_{2}+\frac{2}{3} \mu_{3}\right)$.

(1.3) The slope of I satisfies $-\frac{1}{3} \mu_{1}+\frac{2}{3} \mu_{2}+\frac{2}{3} \mu_{3}<\mu(I) \leqslant \mu_{3}$. Then $\left(E_{0}, \Phi_{0}\right)$ is the following Hodge bundle of type $(1,1,1)$ :

$$
\left(E_{0}, \Phi_{0}\right)=\left(E_{1} \oplus I \oplus\left(E / E_{1}\right) / I,\left(\begin{array}{ccc}
0 & 0 & 0 \\
\varphi_{21} & 0 & 0 \\
0 & \varphi_{32} & 0
\end{array}\right)\right) .
$$

Here $\varphi_{21}$ and $\varphi_{32}$ are induced from $\Phi$. The associated graded vector bundle is $E_{0}=\operatorname{Gr}\left(E_{0}\right)=E_{1} \oplus\left(E / E_{1}\right) / I \oplus I$ and its Harder-Narasimhan type is $\operatorname{HNT}\left(E_{0}\right)=\left(\mu_{1}, \mu_{2}+\mu_{3}-\mu(I), \mu(I)\right)$.

(1.4) The slope of I satisfies $\mu(I)=\mu_{2}$. Then the strict inequality $\mu_{3}<\mu_{2}$ holds, the line bundle $I=E_{2} / E_{1}$, and $\left(E_{0}, \Phi_{0}\right)$ is the following Hodge bundle of type $(1,1,1)$ :

$$
\left(E_{0}, \Phi_{0}\right)=\left(E_{1} \oplus E_{2} / E_{1} \oplus E / E_{2},\left(\begin{array}{ccc}
0 & 0 & 0 \\
\varphi_{21} & 0 & 0 \\
0 & \varphi_{32} & 0
\end{array}\right)\right),
$$

where $\varphi_{32}$ is induced from $\Phi$. The associated graded vector bundle is $E_{0}=$ $\operatorname{Gr}\left(E_{0}\right)=\operatorname{Gr}(E)$.

(2) Suppose that $\mu_{2}>\mu$. Then $\mu_{1} \geqslant \mu_{2}>\mu_{3}$, the subbundle $N \subset E_{2}$ defined in (4.2) is a line bundle, and one of the following alternatives holds. 
(2.1) The slope of $N$ satisfies $\mu_{1}+\mu_{2}-\mu_{3}-(2 g-2) \leqslant \mu(N)<\mu$. Then $\left(E_{0}, \Phi_{0}\right)$ is the following Hodge bundle of type $(2,1)$ :

$$
\left(E_{0}, \Phi_{0}\right)=\left(E_{2} \oplus E / E_{2},\left(\begin{array}{cc}
0 & 0 \\
\varphi_{32} & 0
\end{array}\right)\right) .
$$

The associated graded vector bundle is $\operatorname{Gr}\left(E_{0}\right)=\operatorname{Gr}(E)$.

(2.2) The slope of $N$ satisfies $\mu=\mu(N)$. Then $\left(E_{0}, \Phi_{0}\right)$ is the following strictly polystable Hodge bundle:

$$
\left(E_{0}, \Phi_{0}\right)=(N, 0) \oplus\left(E_{2} / N \oplus E / E_{2},\left(\begin{array}{cc}
0 & 0 \\
\varphi_{32} & 0
\end{array}\right)\right)
$$

where $\varphi_{32}$ is induced from $\Phi$. The associated graded vector bundle is $E_{0}=$ $\operatorname{Gr}\left(E_{0}\right)=E_{2} / N \oplus N \oplus E / E_{2}$ and its Harder-Narasimhan type is $\operatorname{HNT}\left(E_{0}\right)=$ $\left(\frac{2}{3} \mu_{1}+\frac{2}{3} \mu_{2}-\frac{1}{3} \mu_{3}, \mu, \mu_{3}\right)$.

(2.3) The slope of $N$ satisfies $\mu<\mu(N) \leqslant \mu_{2}$. Then $\left(E_{0}, \Phi_{0}\right)$ is the following Hodge bundle of type $(1,1,1)$ :

$$
\left(E_{0}, \Phi_{0}\right)=\left(N \oplus E_{2} / N \oplus E / E_{2},\left(\begin{array}{ccc}
0 & 0 & 0 \\
\varphi_{21} & 0 & 0 \\
0 & \varphi_{32} & 0
\end{array}\right)\right)
$$

where $\varphi_{21}$ and $\varphi_{32}$ are induced from $\Phi$. The associated graded vector bundle is $E_{0}=\operatorname{Gr}\left(E_{0}\right)=E_{2} / N \oplus N \oplus E / E_{2}$ and its Harder-Narasimhan type is $\operatorname{HNT}\left(E_{0}\right)=\left(\mu_{1}+\mu_{2}-\mu(N), \mu(N), \mu_{3}\right)$.

(2.4) The slope of $N$ satisfies $\mu(N)=\mu_{1}$. Then the strict inequality $\mu_{1}>\mu_{2}$ holds, the line bundle $N=E_{1}$ and $\left(E_{0}, \Phi_{0}\right)$ is the following Hodge bundle of type $(1,1,1)$ :

$$
\left(E_{0}, \Phi_{0}\right)=\left(E_{1} \oplus E_{2} / E_{1} \oplus E / E_{2},\left(\begin{array}{ccc}
0 & 0 & 0 \\
\varphi_{21} & 0 & 0 \\
0 & \varphi_{32} & 0
\end{array}\right)\right)
$$

where $\varphi_{21}$ and $\varphi_{32}$ are induced from $\Phi$. The associated graded vector bundle is $E_{0}=\operatorname{Gr}\left(E_{0}\right)=\operatorname{Gr}(E)$.

(3) Suppose that $\mu_{2}=\mu$. Then $\mu_{1}>\mu_{2}>\mu_{3}$, the subbundles $I \subset E / E_{1}$ and $N \subset E_{2}$ defined in (4.1) and (4.2) are line bundles, and one of the following alternatives holds.

(3.1) The equivalent conditions $N=E_{1}$ and $I=E_{2} / E_{1}$ hold. Then $\left(E_{0}, \Phi_{0}\right)$ is the following Hodge bundle of type $(1,1,1)$ :

$$
\left(E_{0}, \Phi_{0}\right)=\left(E_{1} \oplus E_{2} / E_{1} \oplus E / E_{2},\left(\begin{array}{ccc}
0 & 0 & 0 \\
\varphi_{21} & 0 & 0 \\
0 & \varphi_{32} & 0
\end{array}\right)\right)
$$

where $\varphi_{21}$ and $\varphi_{32}$ are induced from $\Phi$. The associated graded vector bundle is $E_{0}=\operatorname{Gr}\left(E_{0}\right)=\operatorname{Gr}(E)$. 
(3.2) Otherwise $\left(E_{0}, \Phi_{0}\right)$ is the following strictly polystable Hodge bundle:

$$
\left(E_{0}, \Phi_{0}\right)=\left(E_{1} \oplus E / E_{2},\left(\begin{array}{cc}
0 & 0 \\
\varphi_{31} & 0
\end{array}\right)\right) \oplus\left(E_{2} / E_{1}, 0\right)
$$

where $\varphi_{31}$ is induced from $\Phi$. The associated graded vector bundle is $\operatorname{Gr}\left(E_{0}\right)=$ $\operatorname{Gr}(E)$.

Remark 5.2. The Cases (1.2), (2.2) and (3) cannot happen when the rank and degree are co-prime, i.e., $(3, d)=1$.

Remark 5.3. The condition $\mu_{2}<\mu$ is equivalent to $\mu_{3}>-\frac{1}{3} \mu_{1}+\frac{2}{3} \mu_{2}+\frac{2}{3} \mu_{3}$. In particular the range for $\mu(I)$ in Case (1.2) is non-empty.

Before proceeding with the proof of Theorem 5.1 we deduce a couple of interesting consequences. The theorem shows that, in general, knowledge of the Harder-Narasimhan type of $E$ does not suffice to determine the underlying bundle $E_{0}$ of the limit $\left(E_{0}, \Phi_{0}\right)=$ $\lim _{z \rightarrow 0}(E, z \cdot \Phi)$. However, there are some Harder-Narasimhan types $\left(\mu_{1}, \mu_{2}, \mu_{3}\right)$ for which $E_{0}$ is determined by $E$. We note that, by Proposition 4.2 , one has $0 \leqslant \mu_{1}-\mu_{3} \leqslant 4 g-4$.

Corollary 5.4. Let $(E, \Phi) \in \mathcal{M}(3, d)$ be such that $E$ is an unstable vector bundle of slope $\mu$ and Harder-Narasimhan type $\left(\mu_{1}, \mu_{2}, \mu_{3}\right)$. Assume that $\mu_{1}-\mu_{3}>2 g-2$. Then the limit $\left(E_{0}, \Phi_{0}\right)=\lim _{z \rightarrow 0}(E, z \cdot \Phi)$ is given by (1.4) of Theorem 5.1 if $\mu_{2}<\mu$, by (2.4) of Theorem 5.1 if $\mu_{2}>\mu$, and by (3.1) of Theorem 5.1 if $\mu_{2}=\mu$. In particular $E_{0}=\operatorname{Gr}\left(E_{0}\right)=\operatorname{Gr}(E)$.

Proof. We only have to show that in all the other cases of Theorem 5.1 we have $\mu_{1}-\mu_{3} \leqslant$ $2 g-2$.

In Cases (1.1), (1.2) and (1.3) we have $\mu(I) \leqslant \mu_{3}$ (cf. Remark 5.3). Moreover, by (1) of Proposition 4.3 , we have $\mu_{1}-(2 g-2) \leqslant \mu(I)$. It follows that $\mu_{1}-(2 g-2) \leqslant \mu_{3}$ as desired.

Similarly, in Cases (2.1), (2.2) and (2.3) we have $\mu(N) \leqslant \mu_{2}$ and, by (2) of Proposition 4.3, $\mu_{1}+\mu_{2}-\mu_{3}-(2 g-2) \leqslant \mu(N)$. Hence $\mu_{1}+\mu_{2}-\mu_{3}-(2 g-2) \leqslant \mu_{2}$ which gives the conclusion.

Finally, in Case (3.2) we have $\varphi_{31} \neq 0$ (since otherwise $E$ would be semistable) and hence $\mu_{1}-\mu_{3} \leqslant 2 g-2$.

In a similar vein, we shall next see that certain types of Hodge bundles can only be the limit of a Higgs bundle whose underlying vector bundle has the same Harder-Narasimhan type as that of the Hodge bundle.

Before stating the result we recall (see, e.g., [8] or Hausel-Thaddeus [16]) that fixed points of type $(1,1,1)$ of the form

$$
\left(E_{0}, \Phi_{0}\right)=\left(L_{1} \oplus L_{2} \oplus L_{3},\left(\begin{array}{ccc}
0 & 0 & 0 \\
\varphi_{21} & 0 & 0 \\
0 & \varphi_{32} & 0
\end{array}\right)\right)
$$

are usually parametrised by the numerical invariants

$$
\begin{aligned}
& m_{1}=\operatorname{deg}\left(L_{2}\right)-\operatorname{deg}\left(L_{1}\right)+2 g-2, \\
& m_{2}=\operatorname{deg}\left(L_{3}\right)-\operatorname{deg}\left(L_{2}\right)+2 g-2,
\end{aligned}
$$


subject to the conditions

$$
\begin{aligned}
m_{i} & \geqslant 0, \quad i=1,2, \\
2 m_{1}+m_{2} & <6 g-6, \\
m_{1}+2 m_{2} & <6 g-6, \\
m_{1}+2 m_{2} & \equiv 0 \quad(\bmod 3) .
\end{aligned}
$$

For our purposes it is more natural to translate to the invariants $\left(l_{1}, l_{2}, l_{3}\right)$ with $l_{i}=$ $\mu\left(L_{i}\right)=\operatorname{deg}\left(L_{i}\right)$ (subject to the condition $\left.l_{1}+l_{2}+l_{3}=3 \mu\right)$. We then have corresponding components $F_{\left(l_{1}, l_{2}, l_{3}\right)}$ of the fixed locus and the invariants $\left(l_{1}, l_{2}, l_{3}\right)$ are subject to the constraints

$$
\begin{aligned}
& l_{i+1}-l_{i}+2 g-2 \geqslant 0, \quad i=1,2, \\
& \frac{1}{3} l_{1}+\frac{1}{3} l_{2}-\frac{2}{3} l_{3}>0, \\
& \frac{2}{3} l_{1}-\frac{1}{3} l_{2}-\frac{1}{3} l_{3}>0 .
\end{aligned}
$$

Corollary 5.5. Let $\left(E_{0}, \Phi_{0}\right)=\left(L_{1} \oplus L_{2} \oplus L_{3},\left(\begin{array}{ccc}0 & 0 & 0 \\ \varphi_{21} & 0 & 0 \\ 0 & \varphi_{32} & 0\end{array}\right)\right)$ be a Hodge bundle of type $(1,1,1)$ with $\mu\left(L_{1}\right)-\mu\left(L_{3}\right)>2 g-2$. Then $\mu\left(L_{1}\right)>\mu\left(L_{2}\right)>\mu\left(L_{3}\right)$ and any $(E$, $\Phi)$ such that $\lim _{z \rightarrow 0}(E, z \cdot \Phi)=\left(E_{0}, \Phi_{0}\right)$ satisfies $E_{0}=\operatorname{Gr}\left(E_{0}\right)=\operatorname{Gr}(E)$.

Proof. It is easy to see that polystability of $\left(E_{0}, \Phi_{0}\right)$ and the condition $\mu\left(L_{1}\right)-\mu\left(L_{3}\right)>$ $2 g-2$ together imply that $\varphi_{21}$ and $\varphi_{32}$ non-zero.

Inspecting the various cases of Theorem 5.1 we see that only in cases (1.4), (2.4) and (3.1) the limit is a Hodge bundle of type $(1,1,1)$ with $\mu\left(L_{1}\right)-\mu\left(L_{3}\right)>2 g-2$. The conclusion follows since in these cases $E_{0}=\operatorname{Gr}\left(E_{0}\right)=\operatorname{Gr}(E)$.

The two previous corollaries lead to an identification between Shatz and BiałynickiBirula strata in some cases. Recall that $U_{\left(l_{1}, l_{2}, l_{3}\right)}^{+}$denotes the Białynicki-Birula stratum of Higgs bundles whose limits lie in $F_{\left(l_{1}, l_{2}, l_{3}\right)}$ and that $U_{\left(l_{1}, l_{2}, l_{3}\right)}^{\prime}$ denotes the Shatz stratum of Higgs bundles whose Harder-Narasimhan type is $\left(l_{1}, l_{2}, l_{3}\right)$.

Theorem 5.6. Let $\left(l_{1}, l_{2}, l_{3}\right)$ be such that $l_{1}-l_{3}>2 g-2$. Then the corresponding Shatz and Biatynicki-Birula strata in $\mathcal{M}(3, d)$ coincide:

$$
U_{\left(l_{1}, l_{2}, l_{3}\right)}^{\prime}=U_{\left(l_{1}, l_{2}, l_{3}\right)}^{+} .
$$

\subsection{Proof of Theorem 5.1}

For the proof, we adopt the complex analytic point of view as explained in Section 2.1. Let $\mathcal{E}$ be the $C^{\infty}$ bundle underlying $E$ and consider the pair $\left(\bar{\partial}_{E}, \Phi\right)$ representing $(E, \Phi)$ in the configuration space of all Higgs bundles. Our strategy of proof is to find a family of gauge transformations $g(z) \in \mathcal{G}^{\mathbb{C}}$, parametrised by $z \in \mathbb{C}^{*}$, such that the limit in the configuration space

$$
\left(\bar{\partial}_{E_{0}}, \Phi_{0}\right)=\lim _{z \rightarrow 0}\left(g(z) \cdot\left(\bar{\partial}_{E}, z \cdot \Phi\right)\right)
$$

gives a stable Higgs bundle $\left(E_{0}, \Phi_{0}\right)$. It will then follow that $\left(E_{0}, \Phi_{0}\right)$ represents the limit in the moduli space.

We now need to consider several cases. 


\subsubsection{Proof of Theorem $5.1-$ Case (1)}

Suppose that $\mu_{2}<\mu$. Then, since $\mu_{1}>\mu$, we must have $\mu_{1}>\mu_{2} \geqslant \mu_{3}$. It follows from

(1) of Proposition 4.3 that $I \subset E / E_{1}$ is a line bundle and that $\mu_{1}-(2 g-2) \leqslant \mu(I) \leqslant \mu_{2}$.

We consider two separate cases.

Case A: $\mu_{1}-(2 g-2) \leqslant \mu(I)<-\frac{1}{3} \mu_{1}+\frac{2}{3} \mu_{2}+\frac{2}{3} \mu_{3}$.

We have a short exact sequence $0 \rightarrow E_{1} \rightarrow E \rightarrow E / E_{1} \rightarrow 0$. Let $\mathcal{E}, \mathcal{E}_{1}$ and $\mathcal{E}_{2}$ be the $C^{\infty}$ vector bundles underlying $E, E_{1}$ and $E / E_{1}$, respectively. Then

$$
\mathcal{E} \cong \mathcal{E}_{1} \oplus \mathcal{E}_{2}
$$

and the holomorphic structure on $\mathcal{E}$ is given by the $\bar{\partial}$-operator:

$$
\bar{\partial}_{E}=\left(\begin{array}{cc}
\bar{\partial}_{1} & \beta \\
0 & \bar{\partial}_{2}
\end{array}\right)
$$

where $\bar{\partial}_{1}$ and $\bar{\partial}_{2}$ are $\bar{\partial}$-operators defining the holomorphic structures on $\mathcal{E}_{1}$ and $\mathcal{E}_{2}$, respectively, and $\beta \in A^{0,1}\left(\operatorname{Hom}\left(\mathcal{E}_{2}, \mathcal{E}_{1}\right)\right)$. With respect to the smooth decomposition (5.1), the Higgs field $\Phi \in A^{1,0}(\operatorname{End}(\mathcal{E}))$ takes the form:

$$
\Phi=\left(\begin{array}{ll}
\varphi_{11} & \varphi_{12} \\
\varphi_{21} & \varphi_{22}
\end{array}\right)
$$

Consider, for each $z \in \mathbb{C}^{*}$, the constant gauge transformation $g(z) \in \mathcal{G}^{\mathbb{C}}$ defined by

$$
g(z):=\left(\begin{array}{cc}
1 & 0 \\
0 & z \cdot I
\end{array}\right)
$$

with respect to the decomposition (5.1). Then:

$$
g(z) \cdot(z \cdot \Phi)=g(z)^{-1}(z \cdot \Phi) g(z)=\left(\begin{array}{cc}
z \cdot \varphi_{11} & z^{2} \cdot \varphi_{12} \\
\varphi_{21} & z \cdot \varphi_{22}
\end{array}\right) \rightarrow\left(\begin{array}{cc}
0 & 0 \\
\varphi_{21} & 0
\end{array}\right) \text { when } z \rightarrow 0
$$

and, moreover,

$$
g(z) \cdot \bar{\partial}_{E}=g(z)^{-1} \circ \bar{\partial}_{E} \circ g(z)=\left(\begin{array}{cc}
\bar{\partial}_{1} & z \cdot \beta \\
0 & \bar{\partial}_{2}
\end{array}\right) \rightarrow\left(\begin{array}{cc}
\bar{\partial}_{1} & 0 \\
0 & \bar{\partial}_{2}
\end{array}\right) \text { when } z \rightarrow 0 .
$$

Note that this simple formula for the gauge transformed $\bar{\partial}$-operator is valid because the gauge transformation is constant on $X$. Thus, in the configuration space of all Higgs bundles the $\operatorname{limit}_{z \rightarrow 0} z \cdot(E, \Phi)$ is gauge equivalent to

$$
\left(E_{0}, \Phi_{0}\right)=\left(E_{1} \oplus E / E_{1},\left(\begin{array}{cc}
0 & 0 \\
\varphi_{21} & 0
\end{array}\right)\right)
$$

This Higgs bundle will represent the limit in the moduli space $\mathcal{M}(3, d)$ provided that it is stable.

To show stability, we note that there are three kinds of $\Phi_{0}$-invariant subbundles of $E_{0}$, namely $E_{1} \oplus I, E / E_{1}$, and an arbitrary line bundle $L \subset E / E_{1}$. We deal with each case in turn: 
1. The subbundle $E_{1} \oplus I \subset E_{1} \oplus E / E_{1}$. By hypothesis $\mu(I)<-\frac{1}{3} \mu_{1}+\frac{2}{3} \mu_{2}+\frac{2}{3} \mu_{3}$ which is equivalent to $\mu\left(E_{1} \oplus I\right)<\mu(E)=\mu\left(E_{0}\right)$ as required.

2. The subbundle $E / E_{1} \subset E_{1} \oplus E / E_{1}$. It is immediate from the properties of the Harder-Narasimhan filtration that $\mu\left(E / E_{1}\right)<\mu(E)=\mu\left(E_{0}\right)$.

3. A line subbundle $L \subset E / E_{1}$. From the properties of the Harder-Narasimhan filtration we have that either $E_{2} / E_{1} \subset E / E_{1}$ is maximal destabilizing (if $\mu_{2}<\mu_{3}$ ) or $E / E_{1}$ is semistable (if $\mu_{2}=\mu_{3}$ ). Either way we have that $\mu(L) \leqslant \mu_{2}$. Since $\mu_{2}<\mu=\mu(E)$ by hypothesis, it follows that $\mu(L)<\mu(E)=\mu\left(E_{0}\right)$.

Finally note that, clearly, $\operatorname{Gr}\left(E_{0}\right)=E_{1} \oplus E_{2} / E_{1} \oplus E / E_{2}=\operatorname{Gr}(E)$. Altogether we have seen that, under the given conditions on the slope of $I$, the limiting bundle $\left(E_{0}, \Phi_{0}\right)$ is as stated in Case (1.1) of the theorem.

Case B: $-\frac{1}{3} \mu_{1}+\frac{2}{3} \mu_{2}+\frac{2}{3} \mu_{3} \leqslant \mu(I) \leqslant \mu_{2}$.

Define $Q=\left(E / E_{1}\right) / I$ so that we have a short exact sequence $0 \rightarrow I \rightarrow E / E_{1} \rightarrow Q \rightarrow$ 0 . Let $\mathcal{E}_{1}, \mathcal{I}$ and $\mathcal{Q}$ be the $C^{\infty}$ bundles underlying $E_{1}, I$ and $Q$, respectively, so that we have a $C^{\infty}$-decomposition

$$
\mathcal{E}=\mathcal{E}_{1} \oplus \mathcal{I} \oplus \mathcal{Q}
$$

Recalling that $\mathcal{I}$ comes from $\Phi\left(E_{1}\right) \otimes K^{-1}$, we may write the Higgs field $\Phi$ as:

$$
\Phi=\left(\begin{array}{ccc}
\varphi_{11} & \varphi_{12} & \varphi_{13} \\
\varphi_{21} & \varphi_{22} & \varphi_{23} \\
0 & \varphi_{32} & \varphi_{33}
\end{array}\right)
$$

with respect to the decomposition (5.2). Moreover, the holomorphic structure on $E$ is of the form

$$
\bar{\partial}_{E}=\left(\begin{array}{ccc}
\bar{\partial}_{1} & \beta_{12} & \beta_{13} \\
0 & \bar{\partial}_{2} & \beta_{23} \\
0 & 0 & \bar{\partial}_{3}
\end{array}\right) .
$$

Now, for each $z \in \mathbb{C}^{*}$ take the following constant gauge transformation:

$$
g(z):=\left(\begin{array}{ccc}
1 & 0 & 0 \\
0 & z & 0 \\
0 & 0 & z^{2}
\end{array}\right)
$$

of $\mathcal{E}$ with respect to the decomposition (5.2). Then

$$
\begin{aligned}
g(z) \cdot(z \cdot \Phi) & =g(z)^{-1}(z \cdot \Phi) g(z) \\
& =\left(\begin{array}{ccc}
z \cdot \varphi_{11} & z^{2} \cdot \varphi_{12} & z^{3} \cdot \varphi_{13} \\
\varphi_{21} & z \cdot \varphi_{22} & z^{2} \cdot \varphi_{23} \\
0 & \varphi_{32} & z \cdot \varphi_{33}
\end{array}\right) \longrightarrow\left(\begin{array}{ccc}
0 & 0 & 0 \\
\varphi_{21} & 0 & 0 \\
0 & \varphi_{32} & 0
\end{array}\right) \text { when } z \rightarrow 0
\end{aligned}
$$

and

$$
\begin{aligned}
g(z) \cdot \bar{\partial}_{E} & =g(z)^{-1} \circ \bar{\partial}_{E} \circ g(z) \\
& =\left(\begin{array}{ccc}
\bar{\partial}_{1} & z \cdot \beta_{12} & z^{2} \cdot \beta_{13} \\
0 & \bar{\partial}_{2} & z \cdot \beta_{23} \\
0 & 0 & \bar{\partial}_{3}
\end{array}\right) \rightarrow\left(\begin{array}{ccc}
\bar{\partial}_{1} & 0 & 0 \\
0 & \bar{\partial}_{2} & 0 \\
0 & 0 & \bar{\partial}_{3}
\end{array}\right) \text { when } z \rightarrow 0 .
\end{aligned}
$$


Hence, in the configuration space, $\lim _{z \rightarrow 0} z \cdot(E, \Phi)$ is gauge equivalent to

$$
\left(E_{0}, \Phi_{0}\right)=\left(E_{1} \oplus I \oplus\left(E / E_{1}\right) / I,\left(\begin{array}{ccc}
0 & 0 & 0 \\
\varphi_{21} & 0 & 0 \\
0 & \varphi_{32} & 0
\end{array}\right)\right) .
$$

Now we prove that $\left(E_{0}, \Phi_{0}\right)$ is a semistable Higgs bundle. The $\Phi_{0}$-invariant subbundles of $E_{0}$ are the following:

1. The subbundle $I \oplus\left(E / E_{1}\right) / I \subset E_{0}$. We have that $\mu\left(I \oplus\left(E / E_{1}\right) / I\right)<\mu(E) \Longleftrightarrow$ $\mu\left(E_{1}\right)>\mu(E)$, which holds by properties of the Harder-Narasimhan filtration.

2. The subbundle $\left(E / E_{1}\right) / I \subset E_{0}$. The condition $\mu\left(\left(E / E_{1}\right) / I\right) \leqslant \mu(E)$ is equivalent to $-\frac{1}{3} \mu_{1}+\frac{2}{3} \mu_{2}+\frac{2}{3} \mu_{3} \leqslant \mu(I)$ which holds by assumption.

Consider the situation when $-\frac{1}{3} \mu_{1}+\frac{2}{3} \mu_{2}+\frac{2}{3} \mu_{3}=\mu(I)$; this is the only case in which $\left(E_{0}, \Phi_{0}\right)$ is strictly semistable. Then $Q=\left(E / E_{1}\right) / I$ is a $\Phi$-invariant subbundle with $\mu(Q)=\mu$, and it follows that the polystable representative of the $S$-equivalence class of $\left(E_{0}, \Phi_{0}\right)$ is obtained by setting $\varphi_{32}=0$ in $\Phi_{0}$. This leads to the description given in Case (1.2).

It remains to analyze the Harder-Narasimhan type of $E_{0}$ when $-\frac{1}{3} \mu_{1}+\frac{2}{3} \mu_{2}+\frac{2}{3} \mu_{3} \neq$ $\mu(I)$. There are two situations to consider.

The first situation is when $\mu(I) \leqslant \mu(Q)$. Then the Harder-Narasimhan type of $E_{0}$ is $\operatorname{HNT}\left(E_{0}\right)=\left(\mu\left(E_{1}\right), \mu(Q), \mu(I)\right)$. Hence, using Shatz's theorem [24, Theorem 3] that the Harder-Narasimhan polygon rises under specialization, we conclude that $\mu(I) \leqslant$ $\mu\left(E / E_{2}\right)$. This leads to the description given in Case (1.3).

The second situation is when $\mu(I)>\mu(Q)$. Then the Harder-Narasimhan type of $E_{0}$ is $\operatorname{HNT}\left(E_{0}\right)=\left(\mu\left(E_{1}\right), \mu(I), \mu(Q)\right)$. Hence, from Shatz's theorem we deduce that $\mu(I) \geqslant \mu\left(E_{2} / E_{1}\right)$. But $I \subset E / E_{1}$ so, from the properties of the Harder-Narasimhan filtration, we conclude that in fact $\mu(I)=\mu_{2}$. If $\mu_{3}=\mu_{2}$ it follows that $\mu(I)=\mu(Q)$, contradicting $\mu(I)>\mu(Q)$. Hence $\mu_{3}<\mu_{2}$ and $I \subset E / E_{1}$ is the unique maximal destabilizing subbundle, i.e., $I=E_{2} / E_{1}$ and so Case (1.4) occurs.

This completes the proof of Case (1).

Remark 5.7. The arguments given for Case A above apply word for word to prove Proposition [3.1. except that the argument to show that $\left(E_{0}, \Phi_{0}\right)$ is a semistable Higgs bundle is simpler: indeed, in the rank 2 case, the only $\Phi$-invariant subbundle of $E_{0}$ is $E / E_{1}$. This satisfies $\mu\left(E / E_{1}\right)<\mu(E)=\mu\left(E_{0}\right)$ because the subbundle $E_{1}$ is destabilizing, i.e., $\mu\left(E_{1}\right)>\mu(E)$.

\subsubsection{Proof of Theorem $5.1-$ Case (2)}

Suppose that $\mu_{2}>\mu$. Then, since $\mu_{3}<\mu$, we must have $\mu_{1} \geqslant \mu_{2}>\mu_{3}$. It follows from (2) of Proposition 4.3 that $N \subset E_{2}$ is a line bundle and that $\mu_{1}+\mu_{2}-\mu_{3}-(2 g-2) \leqslant$ $\mu(N) \leqslant \mu_{1}$.

We consider two separate cases.

Case C: $\mu_{1}+\mu_{2}-\mu_{3}-(2 g-2) \leqslant \mu(N)<\mu$.

We have a short exact sequence $0 \rightarrow E_{2} \rightarrow E \rightarrow E / E_{2} \rightarrow 0$. Let $\mathcal{E}, \mathcal{E}_{2}$ and $\mathcal{E}_{3}$ be the $C^{\infty}$ vector bundles underlying $E, E_{2}$ and $E / E_{2}$, respectively. Then $\mathcal{E} \cong \mathcal{E}_{2} \oplus \mathcal{E}_{3}$ and the holomorphic structure on $\mathcal{E}$ is given by a $\bar{\partial}$-operator of the form $\bar{\partial}_{E}=\left(\begin{array}{cc}\bar{\partial}_{2} & \beta \\ 0 & \bar{\partial}_{3}\end{array}\right)$, while 


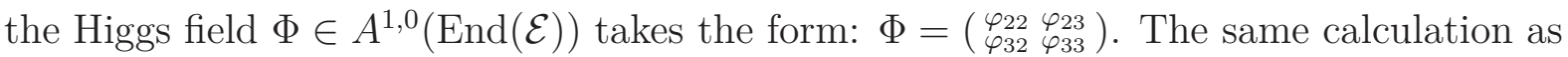
in Case A shows that in the configuration space of all Higgs bundles, $\lim _{z \rightarrow 0} z \cdot(E, \Phi)$ is gauge equivalent to

$$
\left(E_{0}, \Phi_{0}\right)=\left(E_{2} \oplus E / E_{2},\left(\begin{array}{cc}
0 & 0 \\
\varphi_{32} & 0
\end{array}\right)\right)
$$

This Higgs bundle will represent the limit in the moduli space $\mathcal{M}(3, d)$ if it is stable. There are three kinds of $\Phi_{0}$-invariant subbundles to check:

1. The subbundle $N \subset E_{2} \oplus E / E_{2}$. By hypothesis $\mu(N)<\mu=\mu(E)=\mu\left(E_{0}\right)$.

2. The subbundle $E / E_{2} \subset E_{2} \oplus E / E_{2}$. It is immediate from the properties of the Harder-Narasimhan filtration that $\mu\left(E / E_{2}\right)<\mu(E)=\mu\left(E_{0}\right)$.

3. Subbundles $L \oplus E / E_{2} \subset E_{2} \oplus E / E_{2}$ for $L \subset E_{2}$ a line subbundle. From the properties of the Harder-Narasimhan filtration we have that either $E_{1} \subset E_{2}$ is maximal destabilizing (if $\mu_{1}>\mu_{2}$ ) or $E_{2}$ is semistable (if $\mu_{1}=\mu_{2}$ ). Either way we have that $\mu(L) \leqslant \mu_{1}$. It follows that

$$
\begin{aligned}
2 \mu\left(L \oplus E / E_{2}\right) & =\mu(L)+3 \mu-\mu_{1}-\mu_{2} \\
& \leqslant 3 \mu-\mu_{2} \\
& <2 \mu,
\end{aligned}
$$

where we have used the hypothesis $\mu_{2}>\mu$ in the last step. Hence $\mu\left(L \oplus E / E_{2}\right)<$ $\mu=\mu(E)=\mu\left(E_{0}\right)$ as desired.

Finally note that, clearly, $\operatorname{Gr}\left(E_{0}\right)=E_{1} \oplus E_{2} / E_{1} \oplus E / E_{2}=\operatorname{Gr}(E)$. Altogether we have seen that, under the given conditions on the slope of $I$, the limiting bundle $\left(E_{0}, \Phi_{0}\right)$ is as stated in Case (2.1) of the theorem.

Case D: $\mu \leqslant \mu(N) \leqslant \mu_{1}$.

Define $R=E_{2} / N$ so that we have a short exact sequence $0 \rightarrow N \rightarrow E_{2} \rightarrow R \rightarrow 0$. Let $\mathcal{N}, \mathcal{R}$ and $\mathcal{E}_{3}$ be the $C^{\infty}$ bundles underlying $N, R$ and $E / E_{2}$, respectively, so that we have a decomposition of $C^{\infty}$-bundles

$$
\mathcal{E}=\mathcal{N} \oplus \mathcal{R} \oplus \mathcal{E}_{3}
$$

Recalling that $\mathcal{N}$ comes from $\operatorname{ker}\left(\varphi_{21}\right)$, we may write the Higgs field $\Phi$ as:

$$
\Phi=\left(\begin{array}{ccc}
\varphi_{11} & \varphi_{12} & \varphi_{13} \\
\varphi_{21} & \varphi_{22} & \varphi_{23} \\
0 & \varphi_{32} & \varphi_{33}
\end{array}\right)
$$

with respect to the decomposition (5.3). Moreover, the holomorphic structure on $E$ is of the form

$$
\bar{\partial}_{E}=\left(\begin{array}{ccc}
\bar{\partial}_{1} & \beta_{12} & \beta_{13} \\
0 & \bar{\partial}_{2} & \beta_{23} \\
0 & 0 & \bar{\partial}_{3}
\end{array}\right) .
$$


Now take the constant gauge transformation $g(z)=\left(\begin{array}{ccc}1 & 0 & 0 \\ 0 & z & 0 \\ 0 & 0 & z^{2}\end{array}\right)$ of $\mathcal{E}$ with respect to the decomposition (5.3). The same calculation as in Case B shows that in the configuration space $\lim _{z \rightarrow 0} z \cdot(E, \Phi)$ is gauge equivalent to

$$
\left(E_{0}, \Phi_{0}\right)=\left(N \oplus E_{2} / N \oplus E / E_{2},\left(\begin{array}{ccc}
0 & 0 & 0 \\
\varphi_{21} & 0 & 0 \\
0 & \varphi_{32} & 0
\end{array}\right)\right) .
$$

We now prove that $\left(E_{0}, \Phi_{0}\right)$ is a semistable Higgs bundle. The $\Phi_{0}$-invariant subbundles of $E_{0}$ are the following:

1. The subbundle $E / E_{2} \subset E_{0}$. From the properties of the Harder-Narasimhan filtration we have $\mu\left(E / E_{2}\right)<\mu(E)=\mu\left(E_{0}\right)$.

2. The subbundle $E_{2} / N \oplus E / E_{2} \subset E_{0}$. The hypothesis $\mu(N) \geqslant \mu$ is equivalent to $\mu\left(E_{2} / N \oplus E / E_{2}\right) \leqslant \mu=\mu(E)=\mu\left(E_{0}\right)$.

Consider the situation when $\mu(N)=\mu$; this is the only case in which $\left(E_{0}, \Phi_{0}\right)$ is strictly semistable. Then $E_{2} / N \oplus E / E_{2} \subset E_{0}$ is a $\Phi$-invariant subbundle of slope $\mu\left(E_{2} / N \oplus\right.$ $\left.E / E_{2} \subset E_{0}\right)=\mu$, and it follows that the polystable representative of the $S$-equivalence class of $\left(E_{0}, \Phi_{0}\right)$ is obtained by setting $\varphi_{21}=0$ in $\Phi_{0}$. This leads to the description given in Case (2.2).

It remains to analyze the Harder-Narasimhan type of $E_{0}$ when $\mu(N) \neq \mu$. For brevity we write $R=E_{2} / N$. There are two situations to consider.

The first situation is when $\mu(N) \leqslant \mu(R)$. Then the Harder-Narasimhan type of $E_{0}$ is $\operatorname{HNT}\left(E_{0}\right)=\left(\mu(R), \mu(N), \mu_{3}\right)$. Hence, once again using Shatz's theorem, we conclude that $\mu(N) \leqslant \mu_{2}$. This leads to the description given in Case (2.3).

The second situation is when $\mu(N)>\mu(R)$. Then the Harder-Narasimhan type of $E_{0}$ is $\operatorname{HNT}\left(E_{0}\right)=\left(\mu(N), \mu(R), \mu_{3}\right)$. Hence, from Shatz's theorem we deduce that $\mu(N) \geqslant \mu_{1}$. But $N \subset E_{2}$ so, from the properties of the Harder-Narasimhan filtration, we conclude that in fact $\mu(N)=\mu_{1}$. If $\mu_{2}=\mu_{1}$ it follows that $\mu(N)=\mu(R)$, contradicting $\mu(N)>\mu(R)$. Hence $\mu_{2}<\mu_{1}$ and so $N \subset E_{2}$ is the unique maximal destabilizing subbundle, i.e., $N=E_{1}$ and Case (2.4) occurs.

\subsubsection{Proof of Theorem $5.1-$ Case (3)}

Suppose that $\mu_{2}=\mu$. Then, since $E$ is unstable, we must have $\mu_{1}>\mu_{2}>\mu_{3}$. It follows from Proposition 4.3 that the subbundles $I \subset E / E_{1}$ and $N \subset E_{2}$ are line bundles.

Consider the line bundle $N \subset E_{2}$. If $N \neq E_{1}$ we have a non-zero map

$$
N \rightarrow E_{2} / E_{1}
$$

It follows that $\mu(N) \leqslant \mu\left(E_{2} / E_{1}\right)=\mu_{2}=\mu$. Arguing as in Case $\mathrm{C}$ above, we see that in the configuration space of all Higgs bundles, $\lim _{z \rightarrow 0} z \cdot(E, \Phi)$ is gauge equivalent to

$$
\left(E_{0}, \Phi_{0}\right)=\left(E_{2} \oplus E / E_{2},\left(\begin{array}{cc}
0 & 0 \\
\varphi_{32} & 0
\end{array}\right)\right)
$$

and that this is strictly semistable. Moreover, the subbundle $E_{1} \oplus E / E_{2}$ is $\Phi$-invariant and has slope $\mu\left(E_{1} \oplus E / E_{2}\right)=\mu_{2}=\mu$. Hence the polystable representative of the $S$-equivalence class of $\left(E_{0}, \Phi_{0}\right)$ is as stated in Case (3.2). 
Now suppose that $N=E_{1}$. In this case we can argue as in Case D above and see that the limit is as stated in Case (3.1).

In an analogous manner, we see that if $I \neq E_{2} / E_{1}$ the polystable representative of the $S$-equivalence class of $\left(E_{0}, \Phi_{0}\right)$ is as stated in Case (3.2), while if $I=E_{2} / E_{1}$ the limit is as stated in Case (3.1).

Since the Cases (3.1) and (3.2) are mutually exclusive, we see that in fact the conditions $N=E_{1}$ and $I=E_{2} / E_{1}$ are equivalent. This completes the proof of Case (3) and thus the proof of Theorem 5.1 .

\section{References}

[1] L. Álvarez-Cónsul, O. García-Prada, and A. H. W. Schmitt, On the geometry of moduli spaces of holomorphic chains over compact Riemann surfaces, IMRP Int. Math. Res. Pap. (2006), Art. ID 73597, 82.

[2] M.F. Atiyah and R. Bott, Yang-Mills Equations over Riemann Surfaces, Phil. Trans. R. Soc. Lond. Series A, 308 (1982), 523-615.

[3] A. Białynicki-Birula, Some theorems on actions of algebraic groups, Ann. of Math. 98 (1973), 480-497.

[4] S.B. Bradlow, O. García-Prada, and P.B. Gothen, Homotopy Groups of Moduli Spaces of Representations, Topology 47 (2008), 203-224.

[5] M. A. de Cataldo, T. Hausel, and L. Migliorini, Topology of Hitchin systems and Hodge theory of character varieties: the case $A_{1}$, Ann. of Math. (2) 175 (2012), no. 3, 1329-1407.

[6] E. Franco, O. García-Prada, and P. E. Newstead, Higgs bundles over elliptic curves for complex reductive lie groups, preprint, arXiv:1310.2168 [math.AG], 2013.

[7] E. Franco, O. García-Prada, and P. E. Newstead, Higgs bundles over elliptic curves, Illinois J. Math. 58 (2014), no. 1, 43-96.

[8] P.B. Gothen, The Betti Numbers of the Moduli Space of Stable Rank 3 Higgs Bundles on a Riemann Surface, International Journal of Mathematics 5 (1994), no. 6, 861875 .

[9] G. Harder and M.S. Narasimhan, On the Cohomology Groups of Moduli Spaces of Vector Bundles on Curves, Math. Ann. 212 (1975), 215-248.

[10] O. García-Prada and J. Heinloth, The y-genus of the moduli space of $\mathrm{PGL}_{n}$-Higgs bundles on a curve (for degree coprime to n), Duke Math. J. 162 (2013), no. 14, 2731-2749.

[11] O. García-Prada, J. Heinloth, and A. Schmitt, On the motives of moduli of chains and Higgs bundles, J. Eur. Math. Soc. (JEMS) 16 (2014), no. 12, 2617-2668.

[12] T. Hausel, Geometry of Higgs Bundles, PhD thesis, Cambridge University, United Kingdom, 1998. 
[13] Tamás Hausel, Global topology of the Hitchin system, Handbook of moduli. Vol. II, Adv. Lect. Math. (ALM), vol. 25, Int. Press, Somerville, MA, 2013, pp. 29-69.

[14] T. Hausel, E. Letellier, and F. Rodriguez-Villegas, Arithmetic harmonic analysis on character and quiver varieties, Duke Math. J. 160 (2011), no. 2, 323-400.

[15] T. Hausel and F. Rodriguez-Villegas, Mixed Hodge polynomials of character varieties, Invent. Math. 174 (2008), no. 3, 555-624, With an appendix by Nicholas M. Katz.

[16] T. Hausel and M. Thaddeus, Mirror symmetry, Langlands duality, and the Hitchin system, Invent. Math. 153 (2003), 197-229.

[17] T. Hausel and M. Thaddeus, Generators for the Cohomology Ring of the Moduli Space of Rank 2 Higgs Bundles, Proc. London Math. Soc. 88 (2004), no. 3, 632-658.

[18] T. Hausel and M. Thaddeus, Relations in the Cohomology Ring of the Moduli Space of Rank 2 Higgs Bundles, J. Amer. Math. Soc. 16 (2003), 303--327.

[19] N.J. Hitchin, The Self-Duality Equations on a Riemann Surface, Proc. London Math. Soc. 55 (1987), no. 3, 59-126.

[20] N. J. Hitchin, Stable bundles and integrable systems, Duke Math. J. 54 (1987), 91114.

[21] E. Markman, Generators of the cohomology ring of moduli spaces of sheaves on symplectic surfaces, J. Reine Angew. Math. 544 (2002), 61-82.

[22] E. Markman, Integral generators for the cohomology ring of moduli spaces of sheaves over Poisson surfaces, Adv. Math. 208 (2007), no. 2, 622-646.

[23] N. Nitsure, Moduli Space of Semistable Pairs on a Curve, Proc. London Math. Soc. (3) 62 (1991), 275-300.

[24] S.S. Shatz, The Decomposition and Specialization of Algebraic Families of Vector Bundles, Compositio Mathematica 35 (1977), no. 2., 163-187.

[25] O. Schiffmann, Indecomposable vector bundles and stable higgs bundles over smooth projective curves, Ann. Math. 183 (2016), 297--362.

[26] C.T. Simpson, Higgs Bundles and Local Systems, Inst. Hautes Études Sci. Math. Publ. 75 (1992), 5-95.

[27] R.A. Zúñiga-Rojas, Estratificações no espaço móduli dos fibrados de Higgs, Boletim da SPM, Número Especial, ENSPM 2014, Sessão Alunos de Doutoramento, Lisboa (2016), 129-133.

[28] R. A. Zúñiga-Rojas, Homotopy groups of the moduli space of Higgs bundles, Ph.D. thesis, Universidade do Porto, 2015. 